\title{
Thermal and Electromagnetic Stator Vent Design Optimisation for Synchronous Generators
}

\author{
K. Bersch, S. Nuzzo, P. H. Connor, C. N. Eastwick, R. Rolston, M. Galea
}

\begin{abstract}
This paper deals with the stator cooling of an aircooled, synchronous generator with a power rating of $\mathbf{4 0 0}$ kVA, which has been improved by adding two radial vents to the stator. To ensure an optimal vent design, a novel combined thermal and electromagnetic modelling approach is developed. A parametric 3D Conjugate Heat Transfer Computational Fluid Dynamics (CFD) model is used for the thermal modelling. An electromagnetic 2D Finite Element Analysis determined the impact that venting the stator has on the loss distribution. The models are coupled by deriving analytical correlations between the combined vent width and rotor copper, rotor iron and stator iron losses. These correlations are implemented into the optimisation procedure of the parametric CFD model. Five design parameters are optimised simultaneously with the aim of minimising the peak stator winding temperature. The modelling approach was validated experimentally by thermal, torque and mass flow measurements on the benchmark machine, as well as the newly designed prototype.
\end{abstract}

Index Terms - Cooling, electromagnetic modelling, efficiency, CFD, generators, thermal management.

\section{INTRODUCTION}

ELECTRICAL machines (EMs) generate a range of losses that are usually removed via targeted cooling. Recently, more effort is being placed on the thermal design of electrical machines, as the improving cooling analyses and methods enable enhancements in efficiency, life and power density [1], [2].

In the world of EMs, the two main design limits are usually considered to be a combination of electromagnetic and thermal [2]. Whilst the electromagnetic limit is mainly dependent on the geometry and the magnetic properties of the materials, the thermal limit is highly dependent on the ability to efficiently and quickly remove heat from the areas which are most temperature sensitive. In almost all EMs, the primary area to be considered is the machine windings, where advanced thermal management techniques can be employed to improve cooling and in turn efficiency, power density and lifetime [1], [2].

Every family of EMs has its own, common methods for heat removal. Classical, salient-pole synchronous generators (SGs) [3], usually employ a cooling system that performs heat removal from the machine windings, through the use of forced air convection. Due to the physical size and geometrical complexity of such machines, there is usually an extended thermal path from the winding hotspot to the cooling surfaces [4]. For this reason, the main type of heat management for such SGs, especially for higher values of power rating, comprise radial vents. The vents should be

K. Bersch (kevin.bersch@nottingham.ac.uk), P. H. Connor and C. N. Eastwick are with the Fluids and Thermal Engineering Research Group, University of Nottingham, UK.

S. Nuzzo and M. Galea are with the Power Electronics, Machines and Control Research Group, University of Nottingham, UK. placed at strategic locations along the stator stack, to shorten the heat flow path length from the heat generating components to the convective surface, as well as to increase the surface area [5]. Identifying the optimal shape, placement, sizing and number of these vents, requires a thorough understanding of the SG's fluid and thermal behaviour. However, traditionally, the design process of stator vents relied on empirical correlations, rules of thumbs or highly simplified models, coupled with a considerable amount of 'trial and error' work. This has historically led to non-optimal designs for stator venting in large SGs. Venting of stator packs is not typically found in smaller machines, due to the detrimental effects they have on the available output power. With today's efficiency and power density requirements, this is no longer acceptable. Stator winding losses are a function of winding temperature and the convective air flows through vented machines are highly sensitive to geometry changes. To ensure a truly optimal design of stator vents in larger SGs and to potentially exploit the technique even in smaller machines, a new interdisciplinary approach that combines electromagnetic design with advanced high-fidelity fluid and thermal engineering is required.

One of the relatively mature analysis methods, that is a forerunner to the techniques proposed in this paper, is the Lumped Parameter Thermal Network (LPTN). Using LPTNs [6], the designer can describe the SG as a network of thermal resistances and capacitances. However, for design and optimisation purposes, LPTNs require calibration for every design change [7]. A slightly more advanced concept is to use thermal Finite Element Analysis (FEA) [8]. However, thermal FEA tools today are usually limited to the calculation and prediction of thermal conduction problems. To supplement this, pre-set convective boundary conditions are usually set by the machine designer, which are typically a bulk over-simplification, as the local convective behaviour is strongly geometry dependent, especially for vented designs [1]. This step typically reduces the accuracy of the method, which counter-acts the purpose of the optimisation. It is therefore perceived that Computational Fluid Dynamics (CFD) [9] is the only modelling approach to evaluate rotating, turbulent flow, convective and conductive heat transfer simultaneously.

This paper thus focuses on a CFD-approach for the application being considered. As mentioned above, however, CFD alone is not enough to identify the true optimal vent design for a particular SG. An electromagnetic FEA tool is therefore combined with the CFD. To avoid the cost of full two-way numerical linking between the FEA and CFD, this paper presents the novel extraction of a simplified stator vent correlation, specifically for bridging the necessary information between the two physics' solvers, such that the losses generated by the SG can be considered simultaneously with the heat management effects. Ideally, the electromagnetic aspects should be modelled as a 3D problem [10]. However, to

M. Galea (michael.galea@nottingham.edu.cn) is also with the Key Laboratory of More Electrical Aircraft Technology of Zhejiang Province, hosted within the University of Nottingham Ningbo China.

R. Rolston (robert.rolston@cummins.com) is with Cummins Generator Technologies, Peterborough, UK. 
avoid the computational requirements associated with 3D FEA, a 2D FEA is used, whilst incorporating simplified 3D aspects as proposed in [11] and [12].

While electromagnetic analysis and thermal management designs are relatively consolidated techniques, when carried out separately, the linking of these aspects [13] can result in significant advantages in terms of modelling accuracy and effectiveness. On the other hand, both methodologies demand heavy computational resources, predominantly due to the particularly intensive nature of CFD simulations. This paper seeks to show that the benefits that can be reaped from such a combination do overcome the computational cost. Within this paper, a $400 \mathrm{kVA}, 1500 \mathrm{rpm}$ SG has been analysed using a combined FEA and CFD optimisation approach to establish the potential performance gains that adding radial stator vents between laminations may provide. The modelling results were experimentally validated by torque, total mass flow rate, rotor and stator winding temperature measurements on the Benchmark Machine (BM) and the newly designed Vented Prototype (VP), which was manufactured based on the results from the presented multi-physics optimisation process. Elements of numerical optimisation have been performed in the literature [14]-[18], however, this case study builds on these works by exploiting the electromagnetic and CFD combined fluid and conjugate heat transfer analysis for the optimal design of stator vents in woundfield SGs. This paper thus proves that 1) the simplified coupling of the models, 2) the extraction of the EM loss behaviour caused by the inclusion/size/position of the vent, 3) the implementation into an automated CFD conjugate heat transfer optimisation routine, and 4) the definition of User Defined Functions (UDF) for setting the thermal conductivities in the end winding region can lead to significant benefits, even in classical wound-field SGs.

\section{ELECTROMAGNetic ModELLING AND ANALYSIS}

Power losses represent the inherent link between electromagnetic and thermal aspects of any electrical machine. The main modelling aspects from an electromagnetic perspective of the SG studied in this paper are given in this section. The considered industrial platform is a wound-rotor, salient-pole SG featuring 4 poles and 48 slots hosting a star-connected 3-phase, 4 slots-per-pole-per-phase, random-wound armature winding in a double-layer arrangement. The investigated SG is intended for continuous power supply of passive loads, typically of ohmicinductive nature. Examples of utilisation of such generators include small isolated grids, such as boats, hospitals, heavy vehicles, etc.

\section{A. The FEA model and its validation}

Although loss prediction in EMs is historically a difficult task for electromagnetic designers, nowadays much more accurate results than ever before can be achieved through FEA. A 2D model of the SG being considered here was built using the software MagNet ${ }^{\circledR}$, employed and validated in a number of works [12], [19], [20], where all the modeling details were extensively described. Considering the importance for accurate copper and iron loss modelling for this work, these conditions are introduced in the next sections.

\section{1) Copper losses}

In most commercial FEA-based software, the main limitations of a $2 \mathrm{D}$ electromagnetic analysis are related to the fact that it ignores the 3D features of the SG. A number of lumped parameters can be considered in the model through the use of external circuitry emulation [11]. For this investigation, the resistances of the rotor field and stator armature windings have been directly measured, experimentally, at the machine's terminals with an ohmmeter, thus allowing calibration and estimation of the additional resistances included in the FEA model to account for the end winding resistance values.

Thus, 2D transient with motion evaluation can be used to account for the most critical EM characteristics that relate to the end effects without compromising accuracy. An ideal constant current generator is used to feed the SG field winding, whereas lumped ohmic-inductive circuit parameters are employed to simulate a given load according to the output power and the power factor to be analysed. A comparison between FEA and experimental (Exp) copper losses is shown in Fig. 1, where the percentage error at full-load operation is $0.5 \%$, thus validating the accuracy of the simulation models (Sim). The values of the phase currents and resistances were recorded during the experimental tests at the thermal steady-state of the generator (i.e. at the machine operating temperature), which then enables the evaluation of the copper loss contribution. In Fig. 1, losses are shown in p.u., where the experimental total (stator plus rotor) copper loss at full-load and 0.8 power factor are the base value.

\section{2) Iron Losses}

Most FEA packages use the classical Steinmetz model [21], [22] to evaluate iron losses, which can lead to inaccurate results, particularly when high flux density harmonics are present. The method proposed here for the estimation of the iron loss in the SG studied is based on the same Steinmetz model, but it is further extended to all the harmonics [11]. The implemented algorithm considers the rotor surface losses, due to slot harmonics, over the whole amount of iron loss.

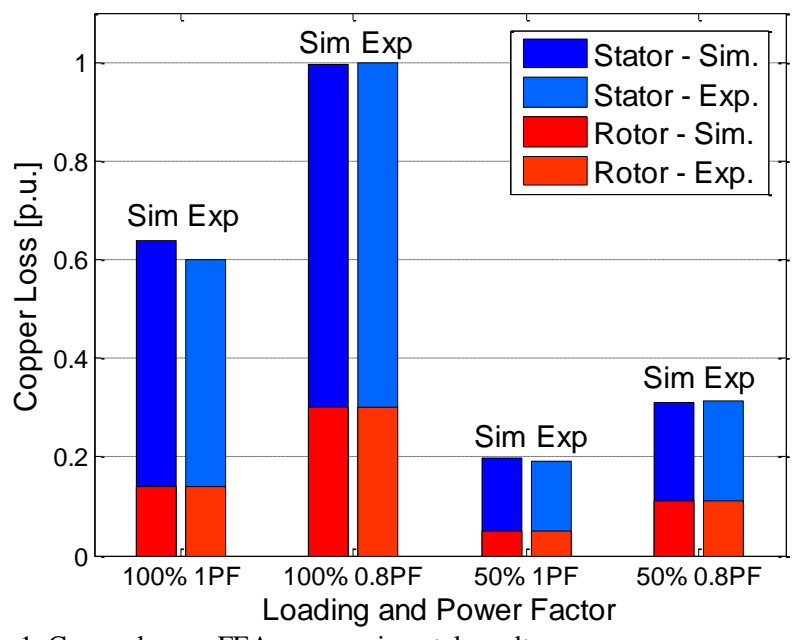

Fig. 1: Copper losses: FEA vs. experimental results

Core losses in SGs are experimentally measured at no-load and are then assumed to be constant for all load levels and power factors. The total core loss is determined as a whole value and does not segregate the rotor, stator or damper cage losses. In Fig. 2, the experimental iron loss value is compared with the SG losses calculated through the algorithm described above. The 
experimental iron loss value is used as the base value to express the iron losses in p.u. Fig. 2 shows that the assumption of having a constant iron loss with loading and power factor is not entirely true. Although they are of an ohmic-resistive nature, the damper cage losses are inherently included in the experimental iron loss value and are therefore considered for the comparison [11]. To be coherent with the experimental results, predicted and measured losses are compared only at no-load. A percentage error of approximately $10 \%$ is observed, highlighting the validity of the proposed computation algorithm.

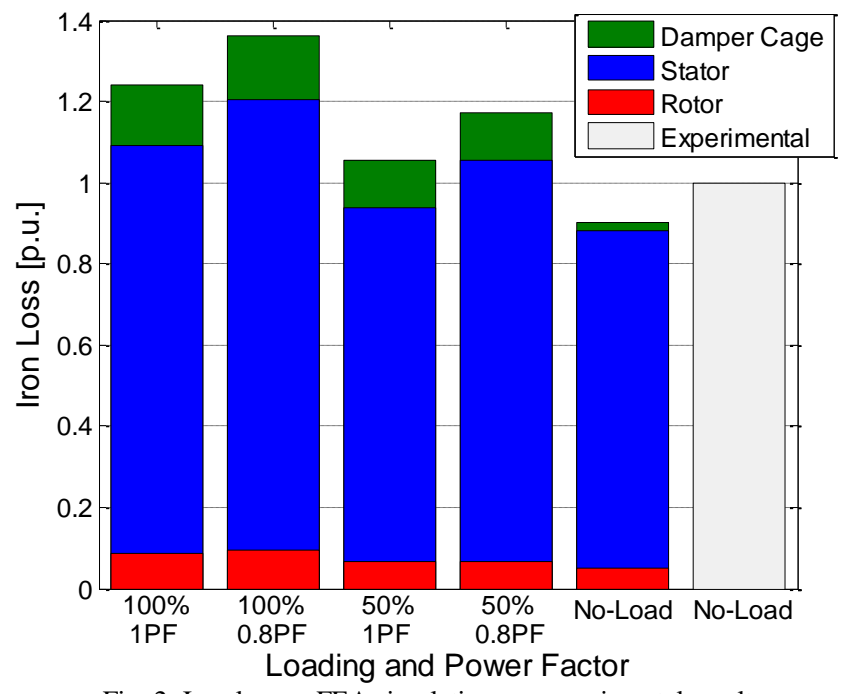

Fig. 2: Iron losses: FEA simulation vs. experimental results

\section{B. The modified FEA model}

Having validated the FEA model (for the BM with no vents), it can then be safely used to analyse machines comprising radial stator ventilating ducts. The optimal design of vents is the main outcome of this paper. Their presence reduces the active material of the stator stack, resulting in a smaller effective core length. This in turn results in a lower power rating of the machine for the same current loading values. An increased field current can be then adopted to maintain the original power values being delivered to the generator's load. However, this measure results in an increase in the field-winding copper loss. Thus, a trade-off study that carefully examines the above arrangements must always be included when doing such modifications to a SG. In fact, using the model described in Section II.A, several machine geometries with different ventilating duct widths, ranging from $2-8 \%$ of the total axial core length, in steps of $1 \%$, are evaluated and their respective losses noted. A simplistic way to to model these vents is to modify the axial length of the machine core. However, the magnetic flux operating in the proximity of these vents will be subject to endeffects, similar to those at the ends of the core stack [23]. While this is inherently a 3D problem, an appropriate measure of this effect can be achieved, even with a 2D model by simply applying the classical Carter factor $k_{C}$ [23]. The equivalent length $L_{e q}$ is approximated by (1), where $L$ is the machine length, including core and ducts, and $n_{v}$ and $b_{v}$ are the number and the width of the ventilating ducts, respectively.

$$
L_{e q}=L-k_{c} n_{v} b_{v}
$$

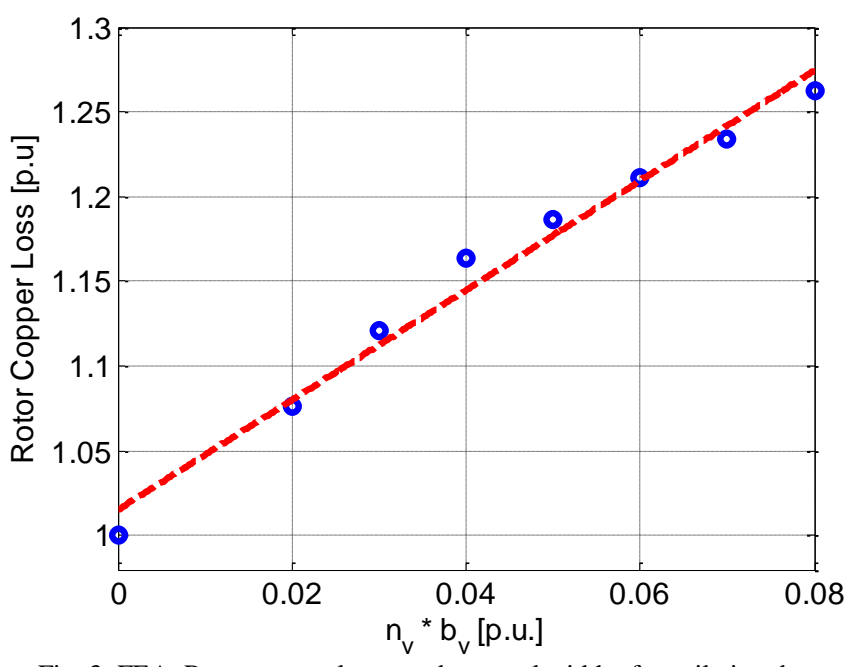

Fig. 3: FEA: Rotor copper loss trend vs. total width of ventilating ducts.

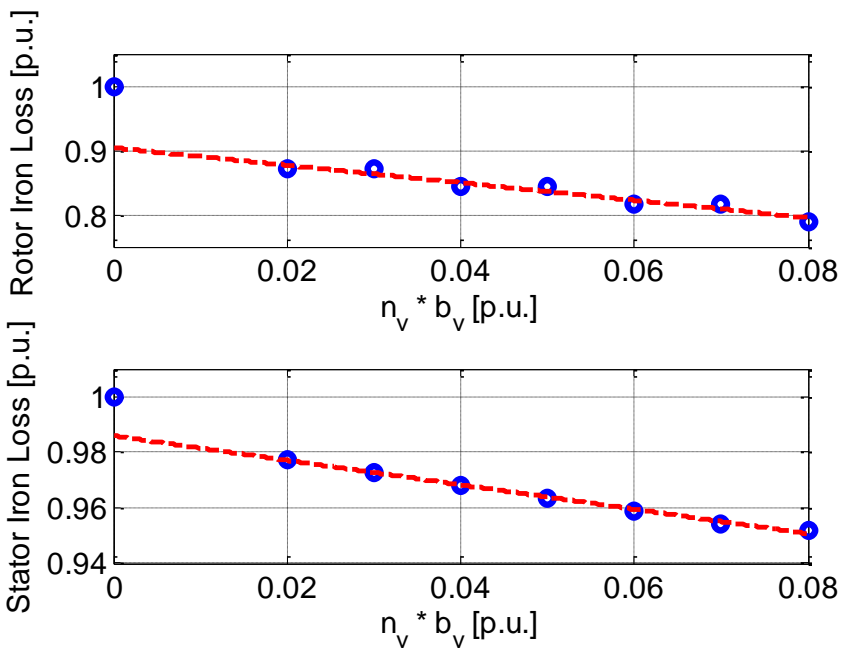

Fig. 4: FEA: Rotor and stator iron loss trend vs. total width of ventilating ducts

Having modified the machine to consider the above effects, the model is run a series of times, for all the investigated vent sizes (or equivalent core lengths), to identify the field current that is required to achieve the same power rating as the original BM. The results of this study are shown p.u. in Fig. 3, where the increasing rotor copper losses, due to the increasing field current, values can be observed. The abscissa $n_{v} * b_{v}=0$ corresponds to the axial length of the original machine (no vents), within which copper losses are thus equal to 1 p.u. in the figure.

Regarding the rotor and stator iron losses (shown p.u. of the rotor and stator iron losses calculated for the original machine), a decreasing linear trend can be observed in Fig. 4. This is because the removal of stator material reduces the overall core volume, which is directly proportional to the iron losses.

The main outcome of this part of the work is that shown in Fig. 3 and Fig. 4. The losses calculated here take into account the inclusion/size/position of the vent as a simplified correlation to feed into the CFD conjugate heat transfer optimisation (which will be described in the following sections), rather than relying on fully coupled numerical modelling, which would be costly and potentially less robust. It is this robust linking between electromagnetic and thermal modelling that will define the appropriateness of the proposed automated optimisation methodology. 


\section{THERMAL MODELLING}

\section{A. Model setup}

The thermal model has been created, solved and optimised using ANSYS Workbench v17.2. The geometry and mesh creation are carried out using the DesignModeler and Meshing packages, which are integrated within Workbench. The solving is carried out using the CFD code ANSYS Fluent. The optimisation procedure was executed using the ANSYS DesignXplorer feature within Workbench, which can be used to make changes to all aspects of the CFD modelling, including the geometry, mesh and solver settings. Combining all steps of the solution and optimisation process in one software package facilitates the creation of a model robust enough to handle the automatic updates during the optimisation process [24], [25].

Modern CFD approaches for EM analyses will typically include not only the fluid flow modelling, but also the conjugate heat transfer behaviour. This refers to the fact that conduction and convection can be solved for all solid parts and surrounding fluid zones inside the machine, simultaneously. This offers a significant improvement in model complexity, detail and accuracy over the alternative LPTN or FEA thermal approaches. The combination of this state-of-the-art CFD analyses with the addition of the electromagnetic modelling within this paper is a noteworthy and novel step forward. Linking the numerical models of the EM FEA with the CFD provides the improved accuracy and detail sought. The novelty lies in extracting the targeted correlation, relating to only the specific EM and thermal parameters affected by the presence of vents, does this without the significant overhead of more blunt bi-directional linking of full 3D EM and CFD numerical analyses. The available computing resource enabled the SG to be surrounded by a cylindrical air domain, which extended beyond the generator casing boundaries. This improves the prediction of the flow behaviour in and out of the machine, which subsequently improves the flow predictions within the machine housing. The modelled fluid domain extends beyond the machine casing by an axial length $5 x$ the core length and a diameter of $8 x$ the stator outer diameter. The outer fluid domain surface is set with a constant pressure outlet boundary condition to allow airflow to pass in and out of the domain. Due to the high complexity of the model, the $\mathrm{SG}$ was assumed to be $180^{\circ}$ rotationally periodic, thus reducing the model size and computation time. This required two simplifications to the actual machine geometry. The flow through the investigated actual generator is driven by a 13-blade fan. This was replaced in the model with 12 blades, which is rotationally periodic in the $180^{\circ}$ model. Similarly, one of the two inlets were moved circumferentially to enable the periodic simplification. Early investigations showed that these simplifications had a negligible influence on the model accuracy (see section $\mathrm{V}$ and [26]). The vented machine geometry is shown in Fig. 5.

The mesh has been created based on the methodology of [27]. Losses were quantified using a combination of experimental measurements and EM FEA (see section II). These were then distributed within the solid components within Fluent as a volumetric heat generation. Mechanical friction in the bearings, electromagnetic stray and stator copper losses were assumed to be constant in all configurations. The rotor copper, rotor and stator iron losses were updated during the model solution based on the total vent width and the correlations shown in Fig. 3 and 4.

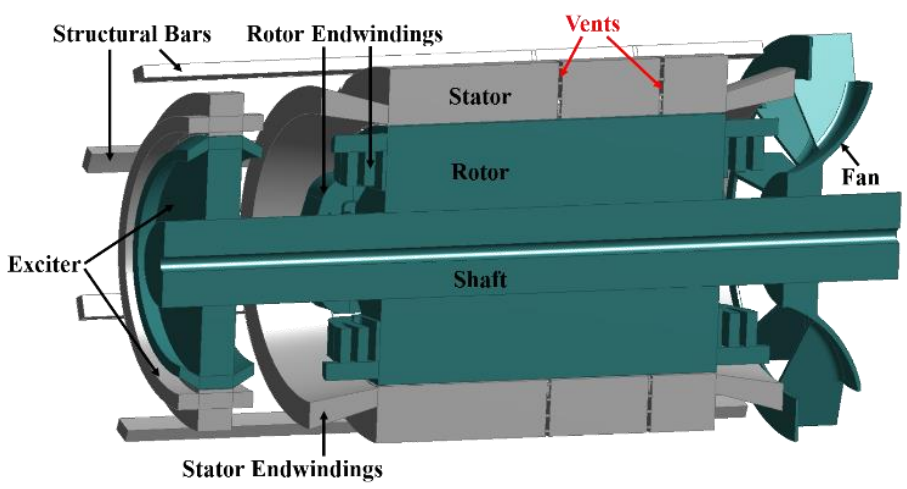

Fig. 5: Vented machine geometry; only solid regions shown for better visibility.

Electrical Machine windings comprise several materials, including copper wires, resin and insulation materials. These materials combine to have a lumped anisotropic effective thermal conductivity behaviour. The effect of this is that the thermal conductivity in the wire direction is considerably higher than the winding thermal conductivity perpendicular to the copper wire orientation. The winding thermal conductivity in the wire direction was assumed to be that of pure copper. The thermal conductivity perpendicular to the wire orientation $k_{l}$ was calculated according to the Hashin and Shtrikman approximation [28], using the individual known material thermal conductivity values of copper $k_{c}$ and the insulating resin $k_{\text {res }}$ according to (2), with $v_{c}$ being the slot fill factor:

$$
k_{l}=\frac{k_{c}\left(1+v_{c}\right) k_{c}+\left(1-v_{c}\right) k_{r e s}}{\left(1-v_{c}\right) k_{c}+\left(1+v_{c}\right) k_{r e s}}
$$

This approach has been used and validated in various works [29]-[31]. The end winding regions are a mix of wire directions. The change in thermal conductivities, due to the direction changes in the physical windings, has been modelled using UDFs in Fluent.

For the stator, a custom material with cylindrical orthotropic conductivity about the z-axis was defined and the axial, radial and circumferential conductivity values were defined dependent on the cell axial location $z$. The endwindings extend straight out of the slots for the first $20 \%$ of their total axial length before they start to bend. Thus, the thermal conductivity was defined equivalent to the core region in this area. From here on, the windings start to bend towards the circumferential direction. A gradual change of thermal conductivity was implemented by assuming a bending radius $r_{b}$ of $50 \%$ of the total axial endwinding length and implementing a linear relationship between the axial $\left(k_{a x}\right)$, radial $\left(k_{\text {rad }}\right)$ and tangential $\left(k_{\text {tan }}\right)$ conductivity and the axial location $z$ according to (3)-(5).

$$
\begin{aligned}
k_{a x}= & \left(1-\frac{z}{r_{b}}\right) k_{c}+\frac{z}{r_{b}} k_{l} \\
& k_{\text {rad }}=k_{l} \\
k_{\text {tan }}= & \left(1-\frac{z}{r_{b}}\right) k_{l}+\frac{z}{r_{b}} k_{h}
\end{aligned}
$$

The bending radius was based on measurements on the BM endwindings in multiple locations. For the final $30 \%$ of the axial extension of the endwindings, the windings were assumed to revolve around the axis of rotation and the conductivity was 
defined to be $k_{l}$ in axial/radial direction and $k_{c}$ in tangential direction.

To model the rotor endwindings, a custom material with orthotropic conductivity was created. Equivalent to the stator, the rotor endwindings extend in the axial direction initially after leaving the core. Hence, the conductivity components for the initial $14 \%$ of the total axial endwinding length were defined equivalent to the core region. Fig.5 shows how the rotor endwindings bend from the $\mathrm{z}$ - towards the $\mathrm{x}$ - or $\mathrm{y}$-direction (depending on the specific winding) afterwards. In the bending region, the conductivity in $\mathrm{x}-\left(k_{x}\right), \mathrm{y}-\left(k_{y}\right)$, z-direction $\left(k_{z}\right)$ are calculated depending on their z-coordinate $z$, the coordinate of the axis the windings are bending towards $(x$ or $y)$ and $r_{E W}=\left(z^{2}+x^{2}\right)$ 0.5 . For the endwindings bending towards the $\mathrm{x}$-direction, the conductivity components in the bending region are calculated according to (6)-(8):

$$
\begin{gathered}
k_{x}=\sqrt{\left(\frac{x}{r_{E W}} k_{l}\right)^{2}+\left(\frac{z}{r_{E W}} k_{c}\right)^{2}} \\
k_{y}=k_{l} \\
k_{z}=\sqrt{\left(\frac{x}{r_{E W}} k_{c}\right)^{2}+\left(\frac{z}{r_{E W}} k_{l}\right)^{2}}
\end{gathered}
$$

After the bending region, the windings are assumed to be aligned with the $y$-axis and the conductivity components are defined as $k_{y}=k_{\mathrm{c}}$ and $k_{x / z}=k_{l}$.

The gradual change in thermal conductivity from high to low and vice versa increases the model robustness and improves the accuracy of the thermal predictions in the endwindings. Additional details about the UDFs and their source code can be found in [32].

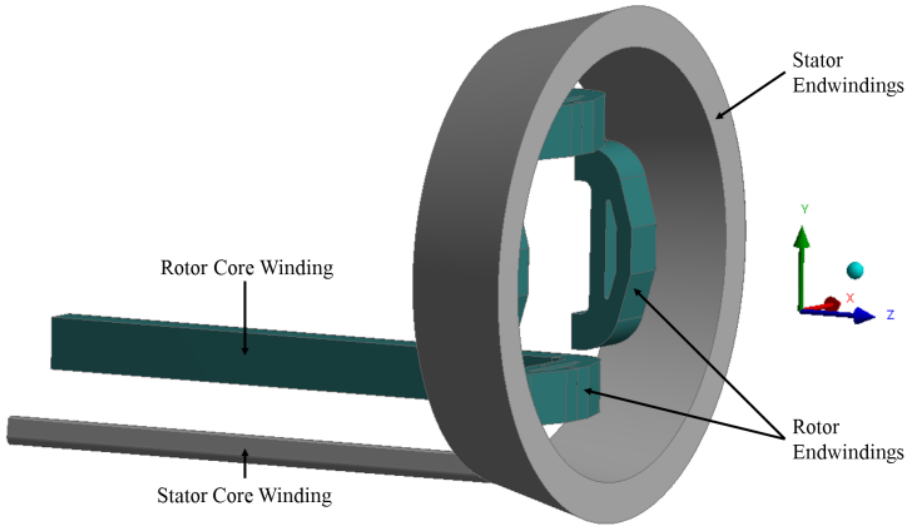

Fig. 6: Rotor and stator endwinding geometry and orientation

The rotor end caps, which are made of plastic, are modelled as an isotropic material with a constant thermal conductivity of $0.3 \mathrm{~W} /(\mathrm{mK})$. All other solid components are made from steel and modelled as isotropic with a constant thermal conductivity of $16 \mathrm{~W} /(\mathrm{mK})$.

Turbulence was modelled using the standard $\mathrm{k}-\varepsilon$ model with enhanced wall treatment. It is commonly used for radial flux machines and has been validated for them by a number of researchers [33]-[35]. The SIMPLE algorithm is employed for the pressure-velocity coupling. The spatial discretisation of all variables is second order accurate. The Multiple Reference Frame technique is used to model rotation. This enables steady-state simulation despite the transient nature of the flow [38]. It is commonly used for EM analyses (see [27], [33]-[35]) over the
Sliding Mesh transient alternative, due to its more practicable lower computational cost, despite lower accuracy.

To reduce the total optimisation time, each case was initialised using a fully converged solution of an early design point (DP). As airflow is more complex and therefore time consuming to solve, beyond the initialisation, the airflow-only is solved for the first 900 iterations. Heat transfer and airflow are then solved simultaneously for 100 further iterations. Convergence was reached in approximately 14 hours per DP on a desktop PC featuring a $3.5 \mathrm{GHz}$ Intel Xeon Hexa-Core and $64 \mathrm{~GB}$ RAM. The solution was considered to be converged, if the residuals and monitored properties of interest, such as windage, mass flow rates, local pressures, maximum and average winding temperatures, were steady. The final residuals ranged from $2 \mathrm{e}-4$ (continuity) to 1e-6 (energy). These are generally considered to be acceptable residual values for whole machine conjugate heat transfer CFD models of electrical machines (see [31], [36], [37]).

The mesh comprises both tetrahedral and hexahedral cells with an average total cell count of 33.3 million. To model the flow through the generator core correctly, the airgap and vents require a very fine mesh. The airgap consists of 20 cells in the radial direction (between rotor and stator). The vents consist of 20 cells in the axial direction (between adjacent stator laminations). On all other surfaces, surface refinements resulting in a $y+$ value in the log-law region (view [38]) were chosen.

\section{B. Optimisation setup}

Simultaneous optimisation of a large number of input parameters is possible for numerical models. However, when considering multiple objectives, in practice, the computational effort of CFD simulations limits the number of parameters to be varied. This is the primary reason as to why multi-physics optimisation for EMs might be considered fairly routine for LPTN modelling, but is highly innovative for CFD linked multi-physics modelling of EMs. Furthermore, CFD solutions are more mesh dependent, and sensitive to poor quality meshing, than FEA. Designing CFD models to be robust enough for fully automated geometry and mesh updates at the required quality for accurate solutions is highly complex and requires an in-depth user knowledge of the flow physics, machine geometry and software. In this paper, it was feasible to optimise five input parameters simultaneously within the available timeframe. Minimising the peak stator temperature was set as the goal for the optimisation.

Early investigations showed adding just a single vent was not sufficient to achieve the targeted stator winding temperature decrease, so a second vent was added to the geometry. Flow rate through the two vents was found to be increased through the addition of a baffle into the stator-casing duct. This increase in airflow showed promise in reducing stator winding temperatures. The axial baffle location was fixed, due to the time constraints of the optimisation process. Initial studies showed that choosing a fixed baffle location at the rear end of the first vent, from the inflow direction, promised to be the best location to reduce the peak stator winding temperature. The baffle protrudes radially inwards from the casing. The five parameters to be varied were identified as follows:

1) Axial location of the $1^{\text {st }}$ vent.

2) Axial location of the $2^{\text {nd }}$ vent.

3) Width of the $1^{\text {st }}$ vent.

4) Width of the $2^{\text {nd }}$ vent.

5) Radial length of the baffle. 
The axial positions of the vents were restricted according to findings of initial investigations and for structural and manufacturing reasons vent widths were limited to $1-4 \%$ of the total core length. Whilst five geometrical parameters were optimised, numerous variable input parameters were defined to robustly result in high quality meshing and to update the variable copper and stator iron losses according to the electromagnetic modelling results generated in section II. The optimisation methodology is based on the findings in [24] and [25]. The Adaptive Single-Objective optimisation algorithm was chosen for this investigation. It combines the creation of response surfaces based on Design of Experiments with a gradient-based optimisation algorithm, reducing the search domain until a converged solution is found (see [39] for further optimisation setup details).

\section{Optimisation Results}

The results presented in the following chapters have been nondimensionalised. Core length, vent location and vent width are normalised by dividing their axial length $z$ by the total core length $L$, i.e. $l_{n}=z / L$. The baffle height $h_{n}$ is normalised by dividing its radial height $H$ by the total radial height of the stator-casing duct $G$, i.e. $h_{n}=H / G$. Local temperatures $T$ are normalised by subtracting the air inlet temperature $T_{\text {in }}$ and dividing by the reference temperature $T_{\text {ref }}$, i.e. $T_{n}=\left(T-T_{i n}\right) / T_{\text {ref }}$. $T_{\text {ref }}$ is defined as the experimentally measured peak stator winding temperature rise above ambient of the BM at full load and 0.8 power factor. Due to time constraints, the optimisation algorithm was stopped after 14 days, i.e. before it reached full convergence. At this point, 25 different configurations were evaluated, and the parameter range was narrowed down.

Table 1 Most promising design points identified during optimisation

\begin{tabular}{|c|c|c|c|c|c|}
\hline & DP 1 & DP 2 & DP 3 & DP 4 & DP 5 \\
\hline Location $1^{\text {st }}$ Vent & 0.47 & 0.50 & 0.51 & 0.54 & 0.50 \\
\hline Location $2^{\text {nd }}$ Vent & 0.82 & 0.80 & 0.86 & 0.84 & 0.80 \\
\hline Width $1^{\text {st }}$ Vent & 0.019 & 0.020 & 0.038 & 0.021 & 0.012 \\
\hline Width $2^{\text {nd }}$ Vent & 0.011 & 0.012 & 0.021 & 0.029 & 0.012 \\
\hline Baffle Lent & 0.32 & 0.41 & 0.46 & 0.14 & 0.41 \\
\hline$\Delta \mathrm{T}_{\max }$ Stator Winding & -0.134 & -0.150 & -0.161 & -0.125 & -0.138 \\
\hline$\Delta \mathrm{T}_{\text {avg }}$ Stator Winding & -0.064 & -0.071 & -0.087 & -0.062 & -0.059 \\
\hline$\Delta \mathrm{T}_{\max }$ Rotor Winding & 0.033 & 0.019 & 0.038 & 0.053 & 0.013 \\
\hline$\Delta \mathrm{T}_{\text {avg }}$ Rotor Winding & 0.045 & 0.040 & 0.060 & 0.063 & 0.034 \\
\hline Total Mass Flow Rate & 0.812 & 0.761 & 0.718 & 0.878 & 0.760 \\
\hline Mass Flow Rate $1^{\text {st }}$ Vent & -0.021 & -0.035 & -0.059 & -0.003 & -0.022 \\
\hline Mass Flow Rate $2^{\text {nd }}$ Vent & -0.033 & -0.036 & -0.060 & -0.060 & -0.037 \\
\hline
\end{tabular}

Due to time constraints, the optimisation algorithm was stopped after 14 days, i.e. before it reached full convergence. At this point, 25 different configurations were evaluated, and the parameter range was narrowed down. The most promising designs (DP 1-4) were identified and discussed with the SG manufacturer. Their key parameters and normalised results are shown in Table 1. The temperature differences are in relation to the BM CFD results. DP 2 was initially identified as the most promising design with differing vent widths. Whilst DP 3 achieves a higher reduction in peak and average stator winding temperatures, the larger vent widths are more problematic structurally, harder to manufacture and increase the rotor temperature more. For simplicity at the prototype manufacturing stage, the design of DP 2 was changed to make both vents equally wide (i.e. $0.012 l_{n}$ ). The CFD model was run with the DP5 new parameters and was shown to still achieve a substantial stator winding temperature reduction, despite the pragmatic design change. Fig. 7 shows a temperature contour plot of a circumferential plane slicing through the stator slot centre, comparing the BM (top) to DP 5 (bottom).

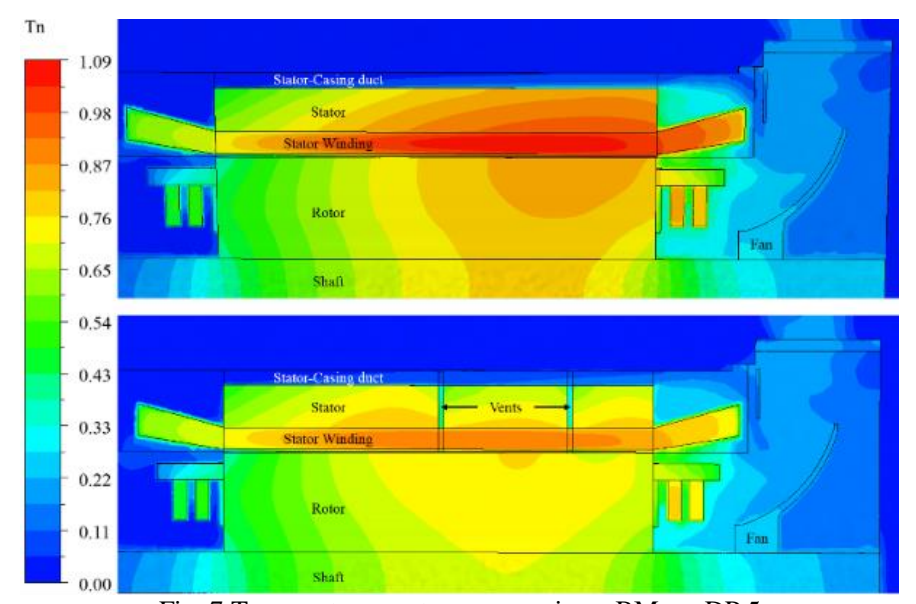

Fig. 7 Temperature contour comparison: BM vs. DP 5

Rotor temperatures are shown to increase with combined vent width (view Table I), which is partly due to the linear increase in rotor copper loss with vent width, as well as the airflow characteristics of this geometry (view section II.B). The BM rotor winding temperatures peak in proximity to the stator winding temperatures, so the addition of stator vents to reduce stator winding temperatures also improve rotor hot spot cooling. This was shown by a simulation of the vented machine with the benchmark heat input. However, the improved rotor cooling is not sufficient to compensate for the increased rotor copper loss and a slight overall rotor winding hotspot temperature increase is seen (see Table 1). Based on the above considerations, a vented stator was built according to the parameters of DP5 and subsequently tested to validate the design methodology.

\section{EXPERIMENTAL VALIDATION}

The modelling results were validated experimentally by torque, total mass flow rate, rotor and stator winding temperature measurements on the BM and the newly designed VP. During the tests, both SGs were driven by an induction motor.

To enable a more consistent comparison between BM and VP, the entire rotating assembly, shown in Fig. 5 and the exciter stator were removed from the BM and inserted into the VP stator for the experimental tests, thus eliminating rotor manufacturing uncertainty as possible test error source.

\section{A. Mass flow rate}

The total mass flow rate was measured by surrounding the machine inlets with a large, airtight box and connecting its only opening to a conical inlet duct. Four pressure tappings were located equidistant circumferentially, downstream of the duct inlet and connected together with plastic tubing. The mass flow rate was calculated from the measured pressure drop according to section 23.4 in BS 848:1 [40]. Further information about the design of the mass flow rate measurement system can be found in [27]. The measurements were taken at no load in $150 \mathrm{rpm}$ intervals in a speed range from 150-1500 rpm. Fig. 8 shows a comparison of CFD and experimentally measured mass flow rates through the $\mathrm{BM}$ and the VP. The mass flow rates were non-dimensionalised, 
by dividing the mass flow rate through the experimental flow rate of the BM at synchronous speed.
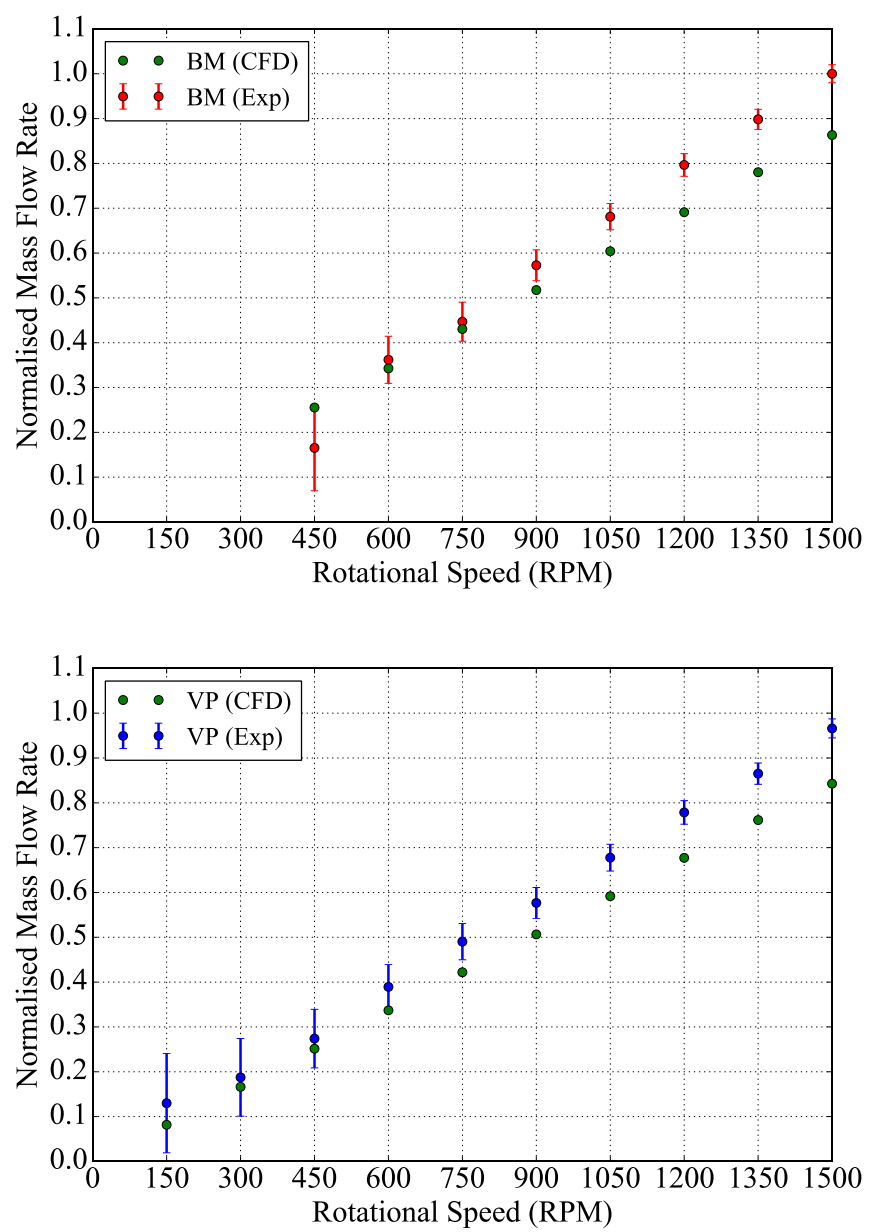

Fig. 8: CFD vs. experimental: mass flow rate through BM (top) and VP (bottom).

The CFD and experimental results show good agreement. At synchronous speed, the difference between CFD and measured mass flow rates amounts to $13.7 \%$ for the $\mathrm{BM}$ and $12.3 \%$ for the VP. The linear relationship between mass flow rate and rotational speed is predicted correctly by the models. As a result of the baffle added to the stator-casing duct, the mass flow rate of the VP is reduced by $3.4 \%$ in comparison to the BM.

\section{B. Torque}

The torque measurements were taken at no-load to avoid uncertainty with segregating the measured torque into electromagnetic torque and mechanical torque produced by friction and windage. The rotor was disconnected from the exciter in order to minimise the torque created by residual magnetism. The measurements were taken in $150 \mathrm{rpm}$ intervals in a rotational speed range from 150-1500 rpm. The torque transducer was located between the drive motor and the coupling.

The measured torque includes the friction generated in the bearings. As this is not considered in the CFD model, it needs to be estimated and subtracted from the experimental torque to make the data comparable. The actual bearing friction torque is complex to calculate and is influenced by a variety of factors, such as the type of bearing, the bearing age, the bearing arrangement, etc. As many of the influencing factors were unknown, a simple analytical approach was chosen to estimate the bearing friction torque $M_{B}$ from the bearing friction coefficient $\mu_{B}$, the radial bearing load $F_{r}$ and the bearing bore diameter $\mathrm{d}_{\mathrm{B}}$ according to (9) [26].

$$
M_{B}=0.5 \mu_{B} F_{r} d_{B}
$$

Fig. 8 shows a comparison of $\mathrm{CFD}$ and experimentally measured torque for the BM and VP. The torque $\mathrm{M}$ was nondimensionalised by dividing the difference of measured torque and the bearing torque $M_{B}$ by the reference torque $M_{\text {ref. }}$ The latter is defined as $\mathrm{M}-\mathrm{M}_{\mathrm{B}}$ at synchronous speed.
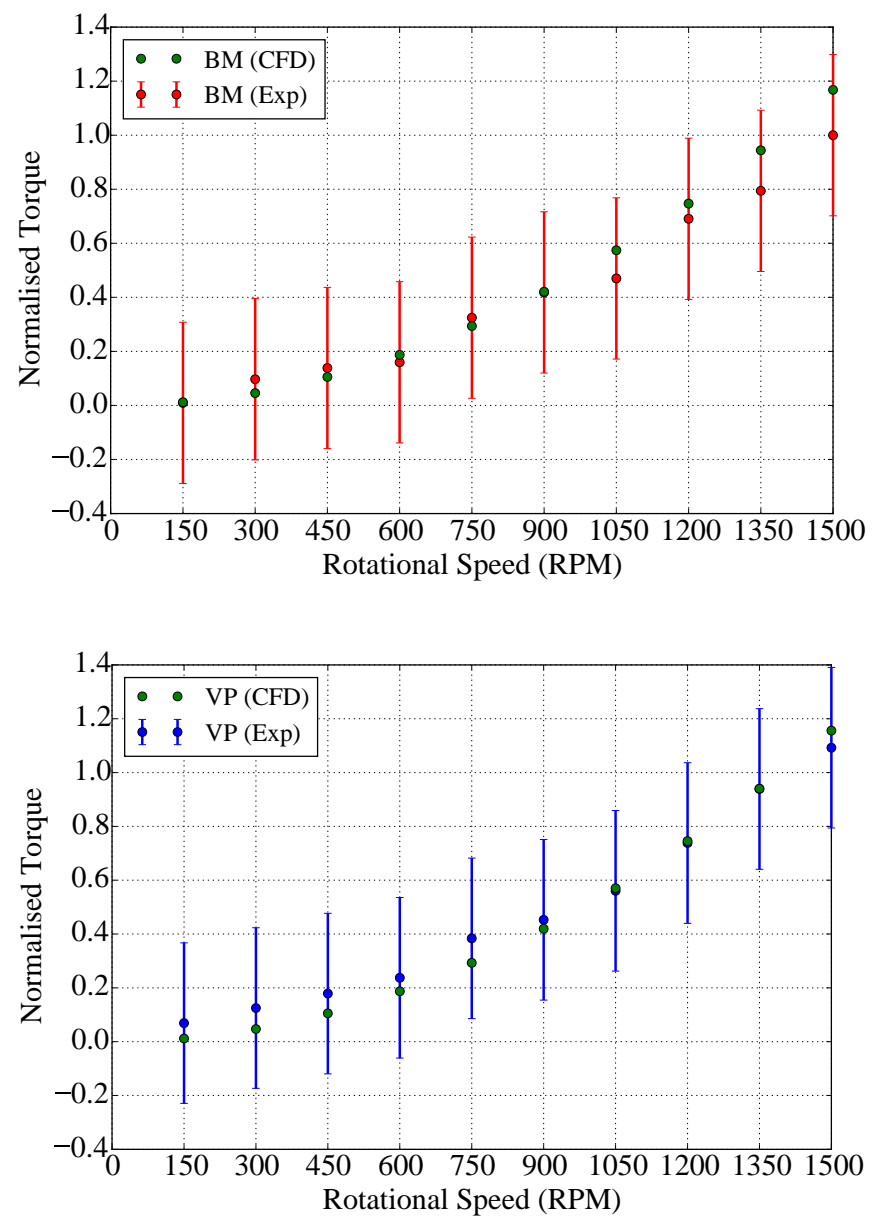

Fig. 9: CFD vs. experimental: Torque of the BM (top) and VP (bottom).

The CFD and experimental results show good agreement. At synchronous speed, the difference between the CFD and experimental torque amounts to $16.8 \%$ for the BM and $6.7 \%$ for the VP. The square relationship between torque and rotational speed is predicted correctly by the models. The test results of the two SGs are within the measurement accuracy, as indicated by the error bars in Fig. 9.

\section{Thermal}

During the generator manufacturing, PT100 Class B Resistance Temperature Detectors (RTDs) were built into the rotor and stator windings in various locations. 42 RTDs were placed in the stator windings, with 30 of them located in the core. They were distributed over 10 equidistant axial locations. 20 RTDs were placed in the rotor windings, 12 of them located in the core and distributed equidistantly over 6 axial locations.

The thermal measurements were taken at rated full load conditions and 0.8 power factor. The generator was connected to the grid during the tests and run for multiple hours to ensure 
thermal steady-state conditions. This methodology enables an accurate prediction of the generators' thermal performance under normal operating conditions. Fig. 10 shows a comparison of the experimentally measured stator winding temperatures in the core between the BM and the VP.

The stator winding temperatures of the VP are significantly reduced all along the core length, confirming that the new vented design improves the stator cooling considerably. The single-phase peak temperature, located at a core length of $0.65 l_{n}$, is reduced by $20.1 \%$. The 3 -phase averaged peak and the average temperatures are reduced by $15.4 \%$ and $12.3 \%$, respectively.

The stator winding temperatures of the BM steadily increase from $0.662 T_{n}$ until their peak located at $0.55 l_{n}$ for the 3-phase average $\left(0.916 T_{n}\right)$ and at $0.65 \mathrm{ln}$ for the single-phase maximum (1.000 $T_{n}$ ). From there on the temperatures decrease towards the Drive End (DE) of the core, with the 3-slot average temperature being $0.852 \mathrm{Tn}$ at the DE.

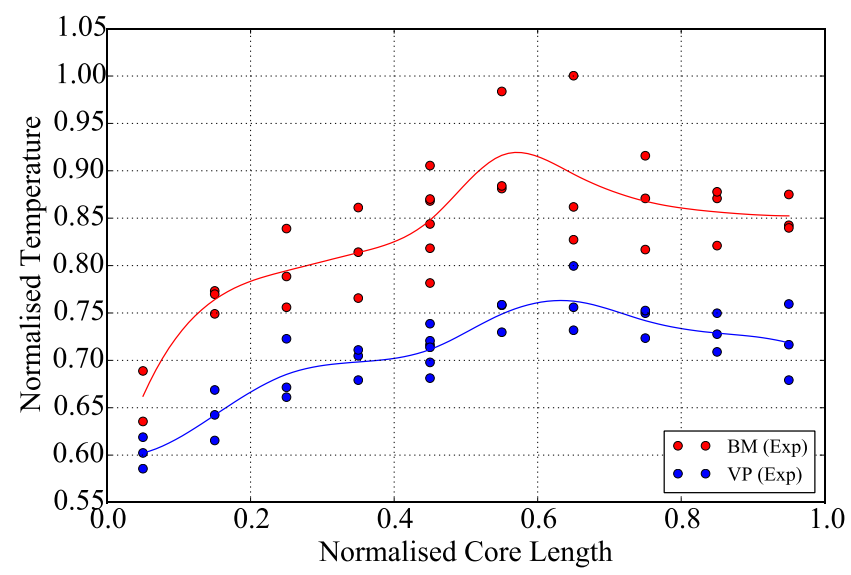

Fig. 10: BM vs. VP experimental results: Stator winding temperatures in the core.

The temperature profile can be explained by the cooling arrangement. The core Non-Drive End (NDE) facing the inlets drawing cold air into the machine, is well cooled. While the air flows along the core, it extracts the heat generated within the generator and warms up. The heat, $\dot{Q}$, that can be extracted from the core decreases with increasing air temperature $T_{\text {air }}$ according to (10), assuming that the surface area $A$ and the heat transfer coefficient $h$ stay constant. Therefore, the stator winding temperature increases until the peak approximately two thirds along the core length. Even though the air temperature continues to increase from here on, the winding temperatures decline as the stator end windings are cooled better than the core windings and heat is conducted from the core to the end windings.

$$
h=\frac{\dot{Q}}{A\left(T_{\text {wall }}-T_{\text {in }}\right)}
$$

Only $35 \%$ of the air entering the generator flows through the rotor-stator gap. The remaining $65 \%$ passes through the statorcasing duct and gets heated up less in comparison to the air passing through the airgap and inter-polar space (view Fig. 6), because of the lower temperature of the stator back iron and the lower flow velocity, resulting in lower HTCs. As the end windings are cooled by all the air entering the machine and their surface area is significantly larger in comparison to the core windings, their cooling is superior. The higher temperatures at the DE in comparison to the NDE can be attributed to the higher temperature of the cooling air, which cumulatively heats up while it flows through the machine core.

The temperature profile of the VP is similar to the BM, increasing from $0.602 T_{n}$ at the NDE to its peak value of $0.800 T_{n}$ (single-phase) or $0.762 T_{n}$ (3-phase average) at $0.65 l_{n}$. From there on, the temperature decreases to $0.718 T_{n}$ at the DE. In addition to the reduction in temperature, the temperature profile of the VP is also more uniform, due to the improved cooling in proximity to the winding hot spot. The VP difference between minimum and maximum temperatures is $0.214 T_{n}$ (single-phase) or $0.160 T_{n}$ (3phase average) compared to $0.365 T_{n}$ (single-phase) or $0.254 T_{n}$ (3-phase average) for the BM.

The stator copper losses implemented into the CFD model were calculated based on the experimentally measured 3-phase averaged stator current and the stator winding resistance measured at thermal steady-state conditions. The rotor copper losses were calculated based on the experimentally measured rotor current and voltage at thermal steady-state conditions. The exciter losses were assumed to be $10 \%$ of the rotor copper losses. The rotor and stator iron losses were implemented based on the EM FEA results discussed in section II. The stray losses were taken from the manufacturer's data sheet and distributed over the main rotor and stator iron and the exciter rotor and stator iron based on volume.

Fig. 10 shows a comparison of CFD and experimentally measured stator winding temperatures in the core for the BM and the VP. The stator winding temperature profiles for both machines are predicted well by the CFD models. The temperature increases from the NDE, peaks in close proximity to the experimental results and declines towards the DE. In comparison to the experimental data, the CFD temperatures are slightly higher. For the BM, the average stator core winding CFD temperature exceeds the 3-phase averaged experimental core winding temperature by $12.0 \%$. The peak temperature difference between CFD and single-phase experimental data is $4.0 \%$. For the VP, the average difference is $13.0 \%$ and the difference in peak temperature amounts to $7.9 \%$. The location of the two vents is evident in the VP CFD temperature profile. Two local minima exist at $0.5 l_{n}$ and $0.8 l_{n}$. They are caused by the windings being directly cooled by the air flowing through the stator vents.

Fig. 11 shows a comparison of CFD and experimentally measured rotor winding temperatures for the BM and the VP. The rotor end windings are displayed in the regions of -0.2-0 $l_{n}$ and 1$1.2 l_{n}$. The experimental rotor winding temperatures increase steadily from the beginning until the end of the core, approximately following a linear trend. For the BM, the peak temperature is $1.012 T_{n}$ at $1.0 l_{n}$, slightly exceeding the peak stator winding temperature. Equivalent to the stator, the rotor end windings are hotter at the DE as the cooling air heats up while it flows through the SG core and stator-casing duct.

The rotor winding temperatures of the VP have marginally increased all along the core length. The average temperature has increased by $2.4 \%$. The maximum increase in temperature was measured to be $3.2 \%$. The temperature rise is caused by the increase in rotor current required to keep the output power constant despite the reduction in effective core length. 


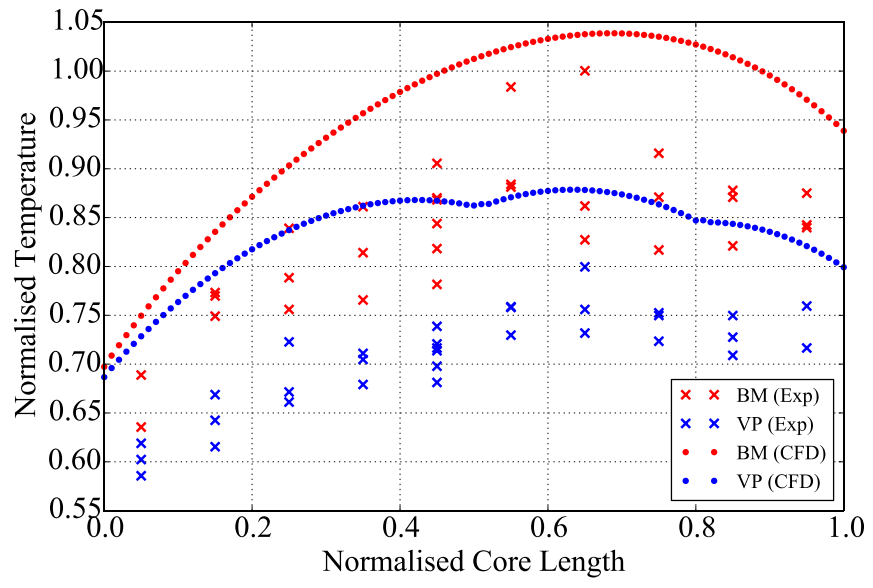

Fig. 11: CFD vs. experimental: Stator winding temperatures in the core

The rotor winding temperatures of the VP have marginally increased all along the core length. The average temperature has increased by $2.4 \%$. The maximum increase in temperature was measured to be $3.2 \%$. The temperature rise is caused by the increase in rotor current required to keep the output power constant despite the reduction in effective core length.

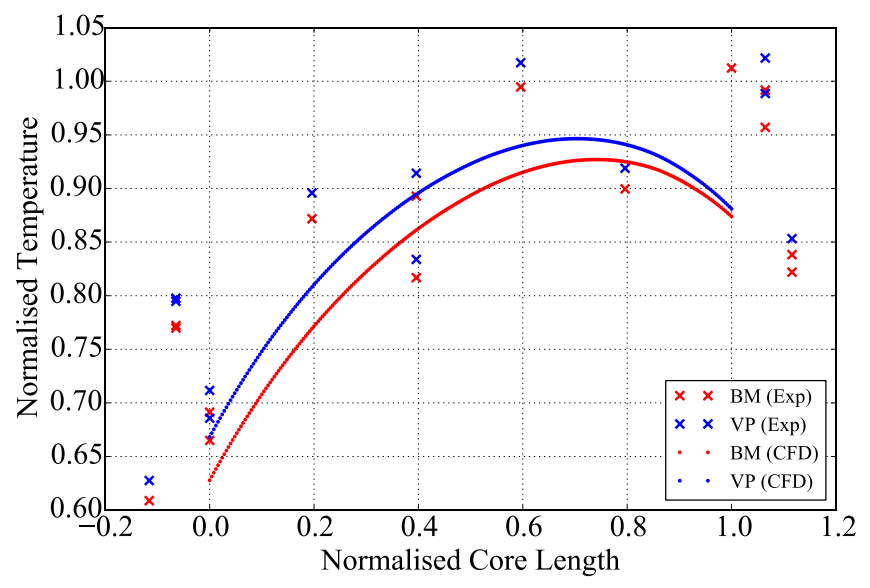

Fig. 12: CFD vs. experimental: Rotor winding temperatures

The CFD and experimental data show good agreement with the CFD temperatures being between the values measured at the two poles equipped with RTDs for the majority of the core length. For the $\mathrm{BM}$, the average CFD rotor winding temperature is $3.6 \%$ below the experimentally measured value. The difference for the VP is $0.1 \%$. The main difference between simulation and experimental results is that the CFD model predicts the rotor winding temperatures to peak at $0.74 l_{n}$, while the peak experimental temperature is located at $1.0 l_{n}$.

\section{CONCLUSION}

This paper has presented an innovative, combined thermal and electromagnetic optimisation procedure for the design of a synchronous generator. The airflow and thermal machine performance is predicted using a 3D Conjugate Heat Transfer CFD model. Electromagnetic 2D FEA was employed to generate analytical correlations between the loss distribution and critical machine design parameters, which were implemented into the thermal optimisation procedure. The developed methodology was applied to a commercial platform to determine the optimal implementation of radial stator vents. Five design parameters were optimised simultaneously with the aim of minimising the peak stator winding temperature: vent locations, vent widths and the height of a baffle added to the stator-casing duct.

Based on the results of this work, the investigated generator was fitted with a new, vented stator and was tested experimentally at rated operating conditions. It was shown that the new design reduces the peak and average stator winding temperatures by 20.1 $\%$ and $12.3 \%$ respectively. A more uniform stator winding temperature profile was achieved. As venting the stator removes active stator iron lamination material and the total core length was kept constant, the rotor current was increased to achieve the same power output. This increased the peak rotor winding temperature by $3.2 \%$ and the average rotor winding temperature by $2.4 \%$. This work has therefore shown that, by exploiting innovative modelling techniques such as combined FEA-CFD optimisations, even cooling methods considered standard and consolidated, such as venting the stator can significantly reduce the stator temperature. The achieved temperature reduction would allow a potential twofold benefit to the machine: 1) the VP SG can be operated at lower temperatures while keeping the same output power as the $\mathrm{BM}$, thus potentially increasing the lifetime of this product, 2) the VP SG can be operated at the same temperature as the BM, thus permitting to increase its output power. For the specific industrial platform studied in this paper, i.e. an SG designed for continuous power supply of passive loads, the first option primarily motivated this work as it was identified by the manufacturer as the most effective methodology to revamp the market value of their product.

\section{REFERENCES}

[1] A. Boglietti, A. Cavagnino, D. Staton, M. Shanel, M. Mueller, and C. Mejuto, "Evolution and Modern Approaches for Thermal Analysis of Electrical Machines," IEEE Trans. Ind. Electron., vol. 56, no. 3, pp. 871-882, 2009.

[2] M. Galea, C. Gerada, T. Raminosoa, and P. Wheeler, "A thermal improvement technique for the phase windings of electrical machines," IEEE Trans. Ind. Appl., vol. 48, no. 1, pp. 79-87, 2012.

[3] S. Nuzzo, M. Galea, C. Gerada and N. Brown, "Analysis, Modeling, and Design Considerations for the Excitation Systems of Synchronous Generators," in IEEE Transactions on Industrial Electronics, vol. 65, no. 4, pp. 2996-3007, April 2018.

[4] P. H. Connor, C. N. Eastwick, S. J. Pickering, C. Gerada, and R. Rolston, "Stator and Rotor Vent Modelling in a MVA rated Synchronous Machine," in 22nd International Conference on Electrical Machines (ICEM 2016), 2016, pp. 571-577.

[5] N. J. Carew and D. H. Freeston, "Fluid Flow Losses in A.C. Generator Stator Ventilating Ducts," Proc. Inst. Mech. Eng., vol. 182, no. 4, pp. 87-95, 1967.

[6] D. G. Dorrel, D. A. Staton, J. Kahout, D. Hawkins, and M. I. McGilp, "Linked Electromagnetic and Thermal Modelling of a Permanent Magnet Motor," in 3rd International Conference on Power Electronics, Machines and Drives (PEMD 2006), 2006, pp. 536-540.

[7] C. Sciascera, P. Giangrande, L. Papini, C. Gerada, and M. Galea, "Analytical thermal model for fast stator winding temperature prediction," IEEE Trans. Ind. Electron., vol. 64, no. 8, pp. 6116-6126, 2017.

[8] R. Deeb, "Thermal Calculation of Permanent Magnet Motors in High Current Technology," Brno University of Technology, 2013.

[9] H. M. Hämäläinen, J. Pyrhönen, J. Nerg, and J. Puranen, “3-D finite element method analysis of additional load losses in the end region of permanentmagnet generators," IEEE Trans. Magn., vol. 48, no. 8, pp. 2352-2357, 2012.

[10] W. Fei, P. C. K. Luk, D. Wu, and B. Xia, "Approximate three-dimensional finite element analysis of large permanent magnet sychronous generators with stator radial ventilating ducts," IECON Proc., pp. 7313-7318, 2013.

[11] S. Nuzzo, M. Degano, M. Galea, C. Gerada, D. Gerada, and N. Brown, "Improved Damper Cage Design for Salient-Pole Synchronous Generators," IEEE Trans. Ind. Electron., vol. 64, no. 3, pp. 1958-1970, 2017.

[12] S. Nuzzo, P. Bolognesi, G. Vakil, D. Fallows, C. Gerada, N. L. Brown, and M. Galea, "A methodology to remove Stator Skew in Small-Medium Size 
Synchronous Generators via innovative damper cage designs," IEEE Trans. Ind. Electron., vol. 66, no. 6, pp. 4296-4307, 2018.

[13] Gołębiowski L., Gołębiowski M., Mazur D. (2015) Thermal Coupling Analysis of the Permanent Magnet Synchronous Generator. In: Gołębiowski L., Mazur D. (eds) Analysis and Simulation of Electrical and Computer Systems. Lecture Notes in Electrical Engineering, vol 324. Springer, Cham

[14] A. Cavagnino, G. Bramerdorfer, and J. Tapia. "Optimization of electric machine Designs - Part I". in IEEE Transactions on Industrial Electronics, 64(12): 9716 - 9720, 2017.

[15] A. Cavagnino, G. Bramerdorfer, and J. Tapia. "Optimization of electric machine Designs - Part II”. in IEEE Transactions on Industrial Electronics, 65(2):1700-1703, 2017.

[16] X. Jannot, J. Vannier, C. Marchand, M. Gabsi, J. Saint-Michel, and D Sadarnac. "Multiphysic modeling of a high-speed interior permanent-magnet synchronous machine for a multiobjective optimal design" in IEEE Transactions on Energy Conversion, 26(2):457-467, 2011.

[17] S. Ulbrich, J. Kopte, and J. Proske. "Cooling fin optimization on a TEFC electrical machine housing using a $2 \mathrm{D}$ conjugate heat transfer model" in IEEE Transactions on Industrial Electronics, 65(2):1711-1718, 2017.

[18] J Buschbeck, M A Vogelsberger, A Orellano, E Schmidt, and M Bazant. "Multi-Physics Optimization of High Power Density Induction Machines for Railway Traction Drives", in 2015 IEEE International Conference on Industrial Technology (ICIT), pages 2656-2661, Seville, Spain, 2015. IEEE

[19] S. Nuzzo, M. Galea, C. Gerada, and N. Brown, "A Fast method for Modelling Skew and its Effects in Salient-Pole Synchronous Generators," IEEE Trans. Ind. Electron., vol. 64, no. 10, pp. 7679-7688, 2017.

[20] S. Nuzzo, P. Bolognesi, C. Gerada, and M. Galea, "Simplified Damper Cage Circuital Model and Fast Analytical-Numerical approach for the analysis of Synchronous Generators," IEEE Trans. Ind. Electron., 2018.

[21] C. P. Steinmetz, "On the Law of Hysteresis," Trans. Am. Inst. Electr. Eng., vol. 9, no. 1, pp. 1-64, 1892

[22] C. P. Steinmetz, "On the Law of Hysteresis (Part II.) and Other Phenomena of the Magnetic Circuit," Trans. Am. Inst. Electr. Eng., vol. 9, no. 1, pp. 619 $758,1892$.

[23] J. Pyrhönen, T. Jokinen, and J. Hrabovcová, Design of Rotating Electrical Machines. Wiley, 2008.

[24] K. Bersch, P. H. Connor, C. N. Eastwick, M. Galea, and R. Rolston, "CFD Optimisation of the Thermal Design for a Vented Electrical Machine," in IEEE Workshop on Electrical Machines Design, Control and Diagnosis (WEMDCD 2017), 2017, pp. 39-44.

[25] K. Bersch, S. Nuzzo, P. H. Connor, C. N. Eastwick, M. Galea, R. Rolston, and G. Vakil, "Combined Thermofluid and Electromagnetic Optimisation of Stator Vent Cooling," in 23rd International Conference on Electrical Machines (ICEM 2018), 2018.

[26] K. Bersch, P. H. Connor, C. N. Eastwick, and M. Galea, "A CFD and experimental investigation into a non-intrusive method for measuring cooling air mass flow rate through a synchronous generator," in 9th International Conference on Power Electronics, Machines and Drives (PEMD 2018), 2018.

[27] P. H. Connor, S. J. Pickering, C. Gerada, C. N. Eastwick, C. Micallef, and C. Tighe, "Computational fluid dynamics modelling of an entire synchronous generator for improved thermal management," IET Electr. Power Appl., vol. 7, no. 3, pp. 231-236, 2013

[28] Z. Hashin and S. Shtrikman, "A Variational Approach to the Theory of the Effective Magnetic Permeability of Multiphase Materials," J. Appl. Phys., vol. 33, no. 10, pp. 3125-3131, 2004.

[29] N. Simpson, R. Wrobel, and P. H. Mellor, "Estimation of equivalent thermal parameters of impregnated electrical windings," IEEE Trans. Ind. Appl., vol. 49, no. 6, pp. 2505-2515, 2013.

[30] L. Idoughi, X. Mininger, F. Bouillault, L. Bernard, and E. Hoang, "Thermal model with winding homogenization and FIT discretization for stator slot," IEEE Trans. Magn., vol. 47, no. 12, 2011.

[31] M. Shanel, "Investigation of Rotor Cooling in Salient Pole Electrical Machines," University of Nottingham, 2002.

[32] Bersch, Kevin (2019) Thermal optimisation of the stator vent design for a synchronous generator. PhD thesis, University of Nottingham.

[33] C. Micallef, "End winding cooling in Electric Machines," University of Nottingham, 2006.

[34] S. J. Pickering, D. Lampard, J. Mugglestone, and M. Shanel, "Using CFD in the Design of Electric Motors and Generators," Comput. Fluid Dyn. Pract., 2001.

[35] J. Mugglestone, D. Lampard, and S. J. Pickering, "Effects of end winding porosity upon the flow field and ventilation losses in the end region of TEFC induction machines," IEE Proc. Electr. Power Appl., vol. 145, no. 5, pp. $423-$ $428,1998$.

[36] H. Versteeg, W. malalasekera, "An Introduction to Computational Fluid Dynamics - The Finite Volume Method. Peasrson Education Limited, 2007.
[37] P. Connor "Computational Fluid Dynamics Modelling of a Synchronous Electric Generator. PhD Thesis, University of Nottingham, UK, 2014

[38] ANSYS Inc., ANSYS Fluent User's Guide Release 17.2. 2016.

[39] ANSYS Inc., ANSYS DesignXplorer User's Guide Release 17.2. 2016.

[40] British Standards Institution, "BS 848-1:2007 Industrial fans - Performance testing using standardized airways," 2007.

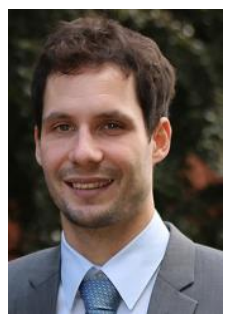

Kevin Bersch received a B.Sc. and M.Sc. degree in Mechanical Engineering and Business Administration from the RWTH Aachen University, Germany, in 2012 and 2015 respectively. He completed his $\mathrm{PhD}$ in the Fluids and Thermal Engineering Research Group of the University of Nottingham in 2019, where he also worked as a research assistant in the Rolls-Royce University Technology Centre in Gas Turbine and Transmission Systems. He is currently working for the Nottingham based engineering consultancy firm Electrical Cooling Solutions, focusing on the thermal optimisation of electrical machines and power electronics using computational and experimental techniques.

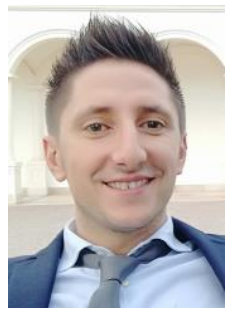

Stefano Nuzzo (S'17-M'18) received the B.Sc. and M.Sc. degrees in Electrical Engineering from the University of Pisa, Pisa, Italy, in 2011 and 2014, respectively. He received his Ph.D. degree in Electrical Engineering in 2018 from the University of Nottingham, Nottingham, U.K, where he is currently working as a Research Fellow within the Power Electronics, Machines and Control (PEMC) Group. Since January 2019, he is also a Research Fellow at the Department of Engineering "Enzo Ferrari" at University of Modena and Reggio, Modena, Italy. His research interests are the analysis, modelling and optimizations of electrical machines, with focus on salient-pole synchronous generators and brushless excitation systems for industrial power generation applications. He is also involved in a number of projects related to the more electric aircraft initiative and associated fields. Dr. Nuzzo is a Member of the IEEE Industrial Electronics Society (IES) and the IEEE Industry Applications Society (IAS). He constantly serves the scientific community as a reviewer for several journals and conference.

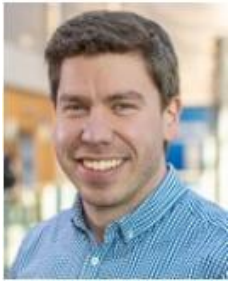

Peter H. Connor received an M.Eng. and Ph.D. from the Department of Mechanical, Materials and Manufacturing Engineering Department, University of Nottingham, UK, in 2009 and 2014 respectively. He is a Senior Research Fellow in the Fluids and Thermal Engineering Research Group and the Power Electronics, Machines and Control Research Group at the University of Nottingham.

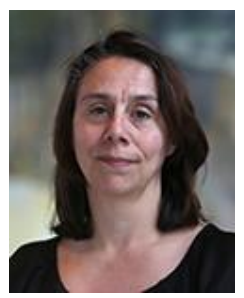

Carol N. Eastwick received her BEng and $\mathrm{PhD}$ in Mechanical Engineering in 1990 and 1995 respectively. She is currently an Associate Professor in the University of Nottingham, having worked on modelling and experimental investigations of thermofluids associated with rotating machinery for over twenty years.

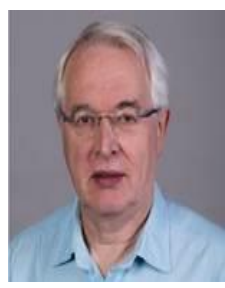

Rob Rolston (received his B.Sc. in Mechanical Engineering from the University of Bristol in 1978 and a Ph.D. from Queen's University of Belfast in 1984. During his career he has worked for GEC Mechanical Engineering Laboratory, United Biscuits Research and Technology Centre, EADS Astrium and for the last three years Cummins Generator Technologies. 


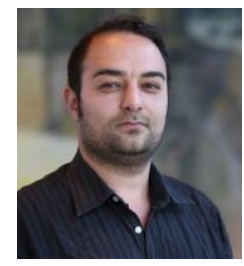

Michael Galea received the Ph.D. degree in electrical machines design in 2013 from the University of Nottingham, Nottingham, U.K. He was appointed as Lecturer in 2014, as Associate Professor in 2018and as Professor in Electrical Machines and Drives in 2019, all with the University of Nottingham. He currently lectures in Electrical Machines and Drives and in Aerospace Systems Integration and manages a number of diverse projects and programmes related to the more/all electric aircraft, electrified propulsion, and associated fields. His main research interests include design and development of electrical machines and drives (classical and unconventional), reliability and lifetime degradation of electrical machines and the more electric aircraft. Michael is a Fellow of the Royal Aeronautical Society and a Senior Member of the IEEE. Michael also serves an Associate Editor for the IEEE Transactions on Industrial Electronics and for the IET Electrical Systems in Transportation. 